injection. Therapy was started in all cases twenty. four hours before inoculation. All five rabbits died on the fifth day and no change in the incubation period or manner of death could be detected.

It would appear that the antibiotics used have no effects on the progress of experimentally induced rabies.

Stack Medical Research Laboratories, J. D. BATE S

Khartoum. March 22.

${ }^{1}$ Nature, 143, 77 (1939).

\section{Hydrolysis of Organic Nitrates}

EARLY work $^{1}$ gives a very confused picture of the reactions which occur in either alkaline or acid hydrolysis of organic esters of nitric acid. The only clearly established point is the occurrence of reactions other than simple fission to alcohol and nitric acid, and the general view was that such side-reactions, including the formation of nitrite, arise from subsequent oxidation of the alcohol by the nitric acid. A more recent study, by Lucas and Hammett ${ }^{2}$, of the solvolysis of tert.-butyl nitrate revealed the concomitant formation of isobutene, while with benzyl nitrate, benzaldehyde and nitrite are also formed.

Analogy with the picture of nucleophilic substitution $\left(S_{N}\right)$ and elimination $(E)$ reactions of alkyl halides, etc., which has resulted from the extensive investigations of Hughes, Ingold and their collaborators ${ }^{3}$, and in which a duality of mechanism (uniand bi-molecular) has been established, suggests that hydrolytic fission of organic nitrates might involve the following three reactions; for simplicity only the bimolecular mechanisms are depicted, although it is evident that, under suitable structural and environmental conditions, the corresponding unimolecular mechanisms may function:

(I) $\underset{R}{\mathrm{X}+\mathrm{CH}_{2}-\mathrm{CH}_{2} \cdot \mathrm{O}} \cdot \stackrel{\mathrm{NO}_{2}}{\mathrm{CH}_{2}} \rightarrow$ $R \cdot \mathrm{CH}_{2} \cdot \mathrm{CH}_{2} \cdot X+\mathrm{NO}_{3}^{\prime}\left(S_{N} 2\right)$ (alcohol + nitrate)

(II) $\prod^{\downarrow} \mathrm{H}-\mathrm{CHR}_{-}^{\downarrow} \mathrm{CH}_{2}-\stackrel{\lceil}{\mathrm{O}} . \mathrm{NO}_{2} \rightarrow$ $\mathrm{CH} R: \mathrm{CH}_{2}+\mathrm{HX}+\mathrm{NO}_{3}^{\prime}(\vec{E} 2)$ (olefine + nitrate)

(III) $\square_{\mathrm{H}} \underset{\mathrm{C}}{\mathrm{CH}}-\mathrm{O}-\mathrm{NO}_{2} \rightarrow$

$R \cdot \mathrm{CH}_{2} \cdot \mathrm{CH}: \overline{\mathrm{O}+\mathrm{H} X}+\mathrm{NO}_{2}{ }^{\prime}\left(E_{C} 2\right)$ (carbonyl + nitrite)

These involve, respectively, attack by the nucleophilic reagent $X\left(=\mathrm{OH}^{\prime}, \mathrm{H}_{2} \mathrm{O}, R \mathrm{OH}\right.$, etc.) at the $\alpha$-carbon, $\beta$-hydrogen or $\alpha$-hydrogen. Theoretically, all these reactions might occur in either alkaline, neutral or acid media.

We are engaged on a detailed kinetic study of these reactions for a large number of organic mono-nitrates in dihute alkaline, neutral and acid aqueous alcoholic solutions, in which the separate reactions I-III have been followed by parallel determinations of $\mathrm{OH}^{\prime}$ (or $\mathrm{H}_{3} \stackrel{+}{\mathrm{O}}$ ), $\mathrm{NO}_{3}{ }^{\prime}, \mathrm{NO}_{2}{ }^{\prime}$, olefine, and carbonyl derivative. One inherent difficulty in determinations by acid-alkali titration is the very small velocity of hydrolysis of simple alkyl nitrates in dilute solution, with the result that, in alkaline media, extensive alkali attack on glass vessels occurs during the course of the experiments, so that the values of $\left[\mathrm{OH}^{\prime}\right]$ obtained bear no relation to the true course of the nitrate hydrolysis. This is a factor which would seem to have been over- looked in the early investigations. This difficulty has been overcome by designing suitable polythene vessels, for which it has been shown that any alkali attack on the vessel is negligibly small during the (prolonged) period over which the hydrolytic reactions have been studied. Under these conditions there is satisfactory agreement between the loss of $\left[\mathrm{OH}^{\prime}\right]$ and the amount of nitrate formed (when only reactions I and II occur), or of nitrate plus nitrite (when all three reactions are superimposed).

Although our data are as yet incomplete, sufficient have been obtained to prove a corresponding agreement between the amount of $\mathrm{NO}_{2}{ }^{\prime}$ and $\mathrm{C}=\mathrm{O}$ derivative formed (reaction III) throughout the course of the reaction. As expected, under the experimental conditions, no oxidation of alcohol is effected by the nitrate ion.

It would appear, therefore, that the formation of olefines, carbonyl derivatives and nitrite does not arise from secondary reactions but from the concomitant occurrence of three main reactions, in two of which (I and II) fission occurs at the C-O linking, and in the third (III) at the $\mathrm{O}-\mathrm{N}$ linking in the nitrate molecule.

A fuller account of this work will be published elsewhere when the investigation is complete.

This note is published by permission of the Chief Scientist, Ministry of Supply.

\section{J. W. BAKER}

D. M. EASTY

Department of Organic Chemistry, University, Leeds 2. March 18

${ }^{1}$ Cf., inter alia, Farmer, J. Chem. Soc., 117, 806 (1920); and references cited there.

${ }^{2}$ J. Amer. Chem. Soc., 64, 1928 (1942).

${ }^{3}$ For summarizing papers, cf. Hughes, Trans. Farad. Soc., 37, 603 (1941); Hughes and Ingold, ibid., 657.

\section{Role of Oxygen in the Photolysis of lodides}

THE decomposition by ultra-violet light of organic compounds containing iodine in solution has been the subject of many investigations, and references to this phenomenon are seattered throughout the photochemical literature. Summaries of the results and conclusions reached by the various investigators have been fairly well dealt with in recent books on the subject ${ }^{1}$. In the majority of cases, the quantum yields of the photochemical process have been determined by the amount of iodine formed in relation to the light absorbed. For the alkyl iodides, at least, these yields show no intelligible variation among themselves, and often the yield for a particular iodide varies from one investigator to another. There always has been a doubt as to whether or not dissolved oxygen was involved in the photolysis ${ }^{2}$, in spite of the fact that many investigators have taken the trouble to distil their materials in vacuo, or to bubble inert gases through the solution before the photolysis. More convineing work along these lines has been undertaken in recent years by Olaerts and Jungers ${ }^{3}$; but it remains to be seen if they have really succeeded in removing the last trace of oxygen from their solutions.

During an investigation of the photodecomposition of iodoacetic acid in hexane, we noticed that the quantum yield for iodine formed $\left(\Phi_{\mathrm{I}_{\mathrm{z}}}\right)$ continued to decrease as more and more precautions were taken to remove the last traces of dissolved oxygen. When no trouble was taken to remove atmospheric oxygen, 